\title{
COMPARISON BETWEEN NANO-HYBRID COMPOSITE AND RESIN REINFORCED GLASS IONOMER IN FLUORIDE RELEASE AND RECHARGE CAPACITY (IN VITRO STUDY)
}

\author{
Sabreen A. Ibrahim ${ }^{1 *}$ BDS, Ahmed A. Mohamed ${ }^{2} P h D$, Adel A. Kamar ${ }^{3} P h D$, \\ Niveen S. Bakry² $P h D$
}

\begin{abstract}
INTRODUCTION: Caries prevention and eradication has been the greatest dentists' challenge world wide. As fluoride has been identified as one of the protective factors that tilt the caries balance towards the positive side. Therefore, fluoride releasing materials were elaborated with the purpose of reducing the incidence of caries.

OBJECTIVES: to compare between the amount of fluoride releasing and recharging capacity of fluoride releasing nano-hybrid composite and resin reinforced glass ionomer.

MATERIALS AND METHODS: Two hundred and seventy two round specimens ( $n=136$ for each material) were used to prepare the required samples. Each 4 specimens immersed in $5 \mathrm{ml}$ of artificial saliva were considered as one sample of total number sixty eight samples ( $\mathrm{n}=34 \mathrm{for}$ each group).The samples were divided randomly into two groups according to the materials used: Group I (experimental): reliaFIL light cure nano-hybrid composite and Group $\Pi$ (control): Riva light cure resin reinforced glass ionomer. The amount of fluoride released in each sample was recorded on day 1,7 and day 15 by using fluoride ion selective electrode. After 15 days each group was divided into 2 subgroups (n=17): Subgroup A: experimental subgroup (treated with ClinproTM white varnish) and Subgroup B: control subgroup (no fluoride varnish treatment). The amount of fluoride re-release was recorded at the same time intervals. Then, the recharge capacity was calculated as the difference in the fluoride release between experimental and the control subgroups.

RESULTS: As for the fluoride release there was a statistical significant difference between two groups ( $\mathrm{P}=0.05)$. However, there was no significant difference between the two groups in the fluoride re-release and recharge capacity $(\mathrm{P}=0.05)$.

CONCLUSIONS: Fluoride releasing nano-hybrid composite had demonstrated a capacity of fluoride release and a recharge capacity comparable to resin reinforced glass ionomer.

KEYWORDS: fluoride release, fluoride recharge, fluoride releasing nano-hybrid composite, resin reinforced glass ionomer, fluoride varnish
\end{abstract}

1- Bachelor of Dentistry, Faculty of Dentistry, Alexandria University, Alexandria, Egypt

2- Professor of Pediatric Dentistry and Dental Public Health Department of Pediatric Dentistry and Dental Public Health, Faculty of Dentistry, University of Alexandria, Alexandria, Egypt

3- Professor of Dental Biomaterials, Department of Dental Biomaterials, Faculty of Dentistry, University of Alexandria, Alexandria, Egypt

*Corresponding author:

E-mail: sabreenalaa89@gmail.com

\section{INTRODUCTION}

Dental caries is one of the most prevalent, chronic, multifactorial and transmissible infectious diseases caused by long term interaction between acid production from bacterial metabolism within the dental biofilm and fermentable carbohydrate (1). While caries is a highly preventable disease that has witnessed a decline in most developed countries in recent years, it continues to remain a global health problem, especially among young children $(2,3)$. Regrettably, secondary caries has been shown in studies worldwide to be the most common reason for the replacement of all types of restorations, regardless of the material used which is destructive for teeth $(4,5)$.

Subsequently, the prime objective of dental treatment nowadays is changed not only for caries restoration but to make an attempt to induce changes in the dental tissues that may resist the initiation of carious process itself (6). A new trend in dental biomaterials science is seeking the improvement of restorative materials properties by the introduction of compounds with antibacterial activity such as chlorhexidine digluconate (CHG) or by the modification with fluoride compounds such as sodium fluoride $(\mathrm{NaF})$ and calcium fluoride (CaF2) which would release fluoride to enhance the remineralization of dental tissue within the cavity and in the environment surrounding the restoration (7).

The role of fluoride in preventing dental caries has been well-documented. It inhibits plaque metabolism and formation, alters its composition and reduces the ability of bacteria to produce large amounts of acid from carbohydrates. Besides this, fluoride inhibits enamel demineralization by being incorporated incrementally as fluorohydroxyapatite crystals on the tooth surface, making the surface more resistant to acid dissolution. In addition to inhibiting demineralization, fluoride increase the speed of the remineralization process by changing the mineral content of early carious lesions causing enhancement of remineralization (8).

There are various restorative materials containing fluoride in their formulation available in the market such as: conventional glass ionomer restorative materials (CGIs), resin modified glass ionomers (RMGIs), and compomers, giomers and resin composites (RC) (9). 
However, it was clinically significant that although advances have been made through different glass ionomer glass powder and placid liquid formulations over the past 40 years, further improvements in the mechanical properties of the current glass ionomers are required to be indicated for the restoration of posterior dentition (10). Moreover, the success rate of resin based composites seems to be declined in the long term due to secondary caries which being attributed mainly to polymerization shrinkage (11). Thus, there has been a growing interest in the development of composites resins with efficient fluoride release abilities and improved physical and mechanical properties to overcome their problem in releasing fluoride when compared to glass ionomers (12).

With the emergence of science of Nanotechnology into the field of dentistry, development of resin materials with more favorable mechanical properties had been developed. Using this technology, a novel fluoride releasing nanohybrid composite (reliaFIL universal light cure composite) was developed aiming to promote better fluoride release and recharge capacity along with improved physical properties (13).

As reliaFIL LC fluoride releasing nano-hybrid composite employed new chemistry, scarce studies examined its efficiency. Therefore, the present study was undertaken with an aim to evaluate the hypothesis that the fluoride releasing nano-hybrid composite (reliaFil LC) can promote fluoride release and recharge capacity after treatment with topical fluoride varnish compared with high fluoride-releasing material (Riva light cure RRGI). The null hypothesis was assumed that there would not be any significant difference between both materials in fluoride release and recharge capacity.

\section{MATERIALS AND METHODS}

This in-vitro experimental study was performed in Department of Pediatric Dentistry and Dental Public Health, Faculty of Dentistry, Alexandria University and in the Institute of Graduate studies and Research, Environmental Studies Department, Alexandria University. The study was performed after receiving approval from the Research Ethical Committee, Faculty of Dentistry, Alexandria University. A sample size of thirty-four samples per group (total samples of sixty eight) was the minimum required sample to detect the smallest standardized effect size according to (Cohen's d) (14) as in figure 1.

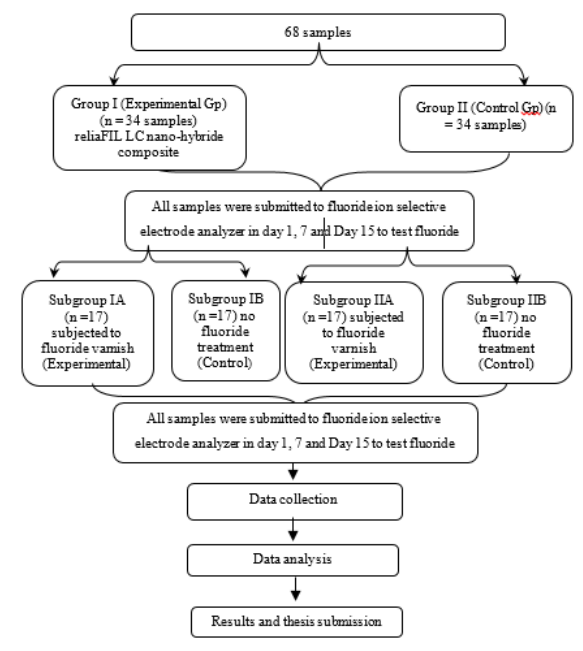

Figure (1): Flow chart.
According to the pilot study performed each four specimens immersed in $5 \mathrm{ml}$ of artificial saliva in plastics vials were considered as one sample. Forming the sixty eight required samples (thirty four samples for each group). Thus, a two hundred and seventy two round specimens (one hundred thirty six for each material) were used to prepare the required samples $(n=68)(15)$. The samples were then divided randomly into two groups according to the materials used: Group I (experimental): reliaFIL light cure nanohybrid composite (reliaFIL LC, shade A2. Advanced Healthcare Ltd (AHL). Kent, England) and Group I (control): Riva light cure resin reinforced glass ionomer (Riva LC RRGIs, shade A2. SDI, Vic, Australia).

The specimens were prepared with a specification of (5 mm diameter $\times 2 \mathrm{~mm}$ thickness) by applying the restorative materials to a specially constructed teflon mold which ensured the standardization of shape and size of each pellet (15). Both materials were prepared according to manufacturer's instructions and cured from top to bottom for 30 seconds for the reliaFIL LC and 20s for Riva LC using led light cure device (Woodpecker Dte Lux V Dental Blue LED Light Cure machine). The specimens in each group were allowed to set for additional 24 hours in a humidifier recipient at $37^{\circ} \mathrm{C}$ with $95 \%$ humidity to simulate the oral environment. Then, all the samples were stored in the incubator for the evaluation tests (16).

\section{Evaluation tests}

\section{Fluoride release}

After 24 hours of immersion, the containers were thoroughly shaken then the specimens were removed, dried and returned into new vials containing $5 \mathrm{ml}$ artificial saliva for the next evaluation on day 7 and day 15 respectively. The fluoride concentration in the artificial saliva was recorded on day 1,7 and day 15 through automated potentiometric titration using a fluoride ion selective electrode (ISE) (Fluoride electrode model 94-09BN, Orion Research Inc. Products gp.529 Main St. Boston MA 02129 USA) for both groups (16). The amount of specific ion (fluorine) contained in artificial saliva was determined by direct potentiometric measurement of the voltage of a galvanic cell (17). Before fluoride analysis, the fluoride ion selective electrode was calibrated by using standard fluoride solution of $0.1,1,10$ and 100 (ppm) to perform the fluoride slope curve. The standard and sample solutions were buffered with a total ionic strength adjustment buffer (TISAB III) at a ratio 1:1 with purpose of adding a chelating reagent to maintain constant $\mathrm{pH}$ and prevent any interference by foreign ions and to decomplex the fluoride ions making them available for measurement (16). The fluoride release concentrations were automatically displayed on the analyzer as millivoltage $(\mathrm{mV})$ readings (17). Millivoltage $(\mathrm{mV})$ readings were entered into the computer using EXCEL software that mathematically established the part per million (ppm) values through the fluoride slope curve of the standard fluoride solution concentration.

- Topical fluoride exposure protocol: After 15 days of initial fluoride release the samples of each group were divided into 2 subgroups of seventeen samples $(n=17)$ : Subgroup A: served as experimental subgroup (in which the specimens were treated by fluoride varnish (Clinpro $^{\mathrm{TM}}$ White Varnish 5\% Sodium Fluoride with Tri-Calcium Phosphate.3M ESPE USAP) for 4 minutes then washed with copious artificial saliva for $10 \mathrm{sec}$ and dried with 
absorbent paper. Each four specimens after fluoride application were again immersed in plastics vials containing $5 \mathrm{ml}$ of fresh artificial saliva and incubated in $37^{\circ} \mathrm{C}$ for 24 hours and Subgroup B: served as control subgroup (no topical fluoride varnish treatment was applied) (16).

- Fluoride re- release evaluation: Artificial saliva was analyzed for fluoride re-release on day 1, 7 and day 15 using fluoride ion selective electrode (16) as previously described.

- Fluoride recharge capacity: Recharge capacity was calculated as the difference in the fluoride release between experimental subgroup and the control subgroup (15).

\section{Statistical analysis}

Data were collected and entered to the computer using SPSS (Statistical Package for Social Science) program for statistical analysis (ver 25.0) (18). Descriptive statistics were displayed as minimum, maximum, median and interquartile range of each group. Normality test was done using Kolmogorov-Smirnov test to determine if data set to be normally distributed. Comparisons of fluoride concentration difference at different time interval were evaluated using Friedman's two-way analysis of variance by ranks. Dunn-Sidak method was used for pair-wise comparison of each two time interval for each group. MannWhitney U test was used for comparison between two study groups at different intervals of time. Wilcoxon Signed Ranks test was used for comparing fluoride concentration before and after recharge. Percent change test was used for analyzing the recharge capacity of each materials during different intervals. Correlation for $\mathrm{p}$ value was carried out using Bonferroni correction for multiple comparisons. Level of significance was set at $p$ value $<0.05$.

\section{RESULTS}

\section{Fluoride release}

The median of fluoride release (ppm) for group I at different time intervals indicated that there was a statistically significant difference between the three intervals $(\mathrm{P}=0.005)$. The median of fluoride release at day 7 was higher than at day 1 and day 15 (Figure 2).

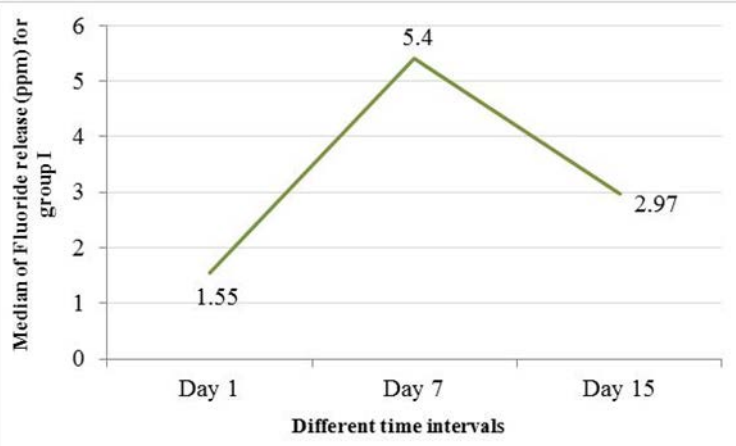

Figure (2): Fluoride release (ppm) for group I (reliaFIL LC nanohybrid composite) at different time intervals.

As for group II, there was only significant difference between the median of fluoride release in day 1 and day 7 $(\mathrm{P}=0.005)$. Whereas, the median of fluoride release in day 7 and day 15 and the median of fluoride release in day 1 and day 15 there was no statistically significant difference $(\mathrm{P}=0064, \mathrm{P}=1.000)$ respectively. The median of fluoride release in day 7 was the highest among the three intervals (Figure 3).

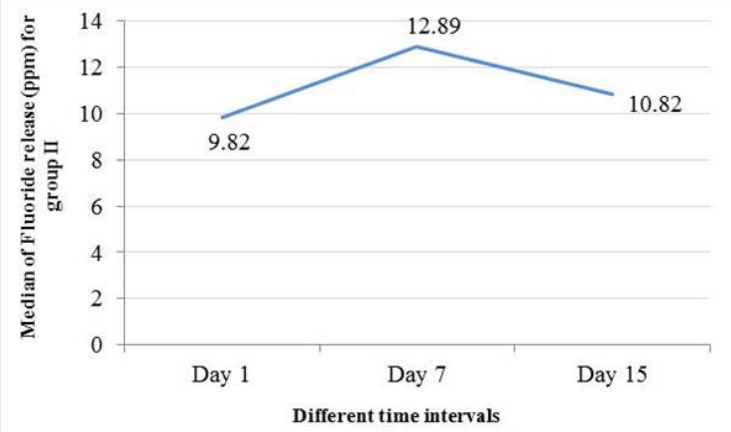

Figure (3): Fluoride release (ppm) for group II (Riva LC RRGIs) at different time intervals.

Comparing the fluoride release between the two groups, there was a statistical significant difference $(\mathrm{P}=0.005)$, where the resin reinforced glass ionomer showed a higher median of fluoride release in all time intervals (Table 1).

Table (1): Comparison between fluoride release (ppm) for the two studied groups I (reliaFIL LC nano-hybrid composite) and group II (Riva LC RRGIs) at different time intervals.

\begin{tabular}{|l|c|c|c|c|}
\hline \multicolumn{1}{|c}{ Material } & \multicolumn{2}{c}{ Day 1 } & \multicolumn{1}{c}{ Day 7 } & \multicolumn{1}{c|}{ Day 15 } \\
\hline \multirow{2}{*}{$\begin{array}{l}\text { ReliaFIL } \\
\text { LC nano- } \\
\text { hybrid } \\
\text { composite }\end{array}$} & Min-Max & $0.95-3.25$ & $1.32-7.53$ & $0.40-4.64$ \\
\cline { 2 - 5 } & Median & 1.55 & 5.40 & 2.97 \\
\hline \multirow{2}{*}{$\begin{array}{l}\text { Riva LC } \\
\text { Resin } \\
\text { reinforced } \\
\text { glass } \\
\text { ionomer }\end{array}$} & IQR & $1.15-2.08$ & $4.36-5.96$ & $2.07-3.30$ \\
\cline { 2 - 5 } & Min-Max & $6.37-27.40$ & $7.56-26.36$ & $6.28-25.47$ \\
\cline { 2 - 5 } & IQdian & 9.82 & 12.89 & 10.82 \\
\hline P value & $8.21-13.40$ & $10.54-$ & $8.26-14.31$ \\
\hline
\end{tabular}

*Statistically significant difference $(\mathrm{p}<0.05)$.

\section{Fluoride re-release}

The median value of fluoride re-release (ppm) for subgroup IA indicated that there was a statistically significant difference between the three intervals $(\mathrm{P}=0.005)$. There was a statistically significant difference between the median of fluoride re-release in day 1,7 and day $15(\mathrm{P}=0.005)$ with the highest fluoride re-release in day 1 . However, there was no statistical significant difference in the median of fluoride rerelease in day 7 and day $15(\mathrm{P}=1.000)$ (Figure 4).

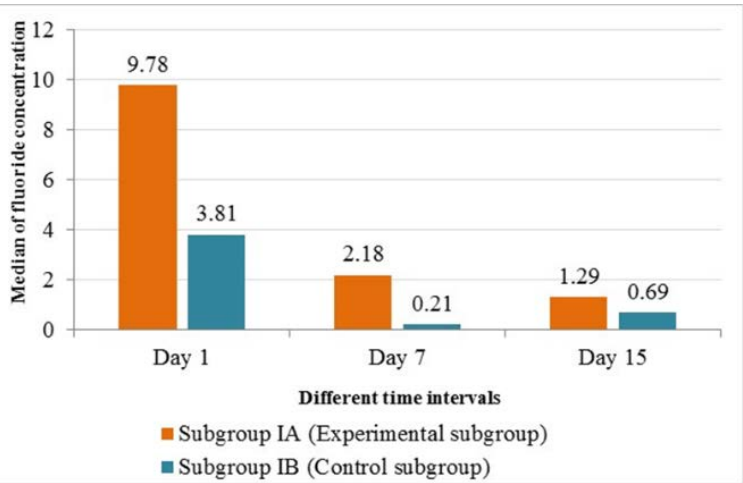

Figure (4): Comparison of fluoride re-release (ppm) between experimental and control subgroups of reliaFIL LC nano-hybrid composite. 
The comparison between the median of fluoride rerelease of subgroup IA and subgroup IB showed that there was a statistically significant difference in fluoride rerelease after recharge for all time intervals $(\mathrm{P}=0.001,0.005$, 0.004 respectively). The median of fluoride concentration demonstrates an increase in the fluoride re-release capacity after recharging with topical fluoride varnish. (Figure 4)

Fluoride re-release (ppm) for subgroup IIA indicated that there was a statistically significant difference between the three intervals $(\mathrm{P}=0.005)$ with the highest re-release in day 1 . The comparison between each two intervals showed that fluoride re-release in day 1 was significantly different than in day 7 and day $15(\mathrm{P}=0.001, \mathrm{P}=0.005)$ respectively. However, fluoride re-release in day 7 was not significantly different than day $15(\mathrm{P}=0.087)$ (Figure 5).

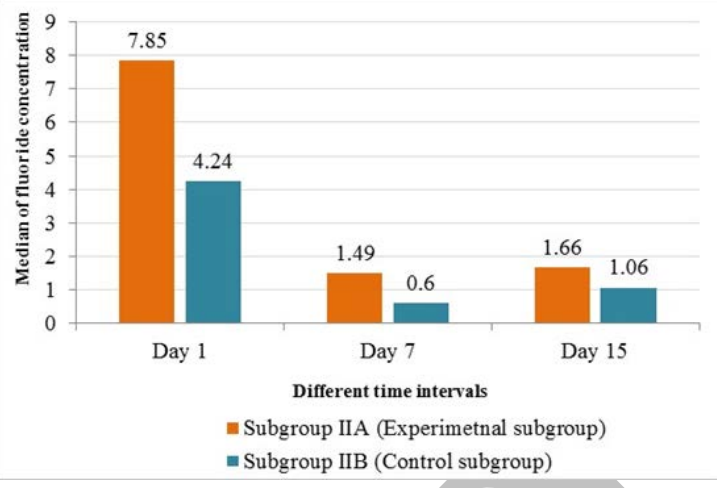

Figure (5): Comparison of fluoride re-release (ppm) between experimental and control subgroups of Riva LC RRGIs.

When comparing between the median of fluoride rerelease of subgroup IIA and subgroup IIB, there was a statistically significant increase in fluoride release after recharge for all time intervals $(\mathrm{P}=0.005,0.001)$. The median of fluoride concentration demonstrates an increase in the fluoride re-release capacity after recharging with topical fluoride varnish. (Figure 5)

3. When comparing between group I and group II after recharging with topical fluoride varnish in each time interval, there was no statistically significant difference in fluoride recharge capacity between the two materials in day 1,7 and day 15 after recharging $(\mathrm{P}=0.237, \mathrm{P}=0.120$, $\mathrm{P}=0.431$ ) respectively (Figure 6 ).

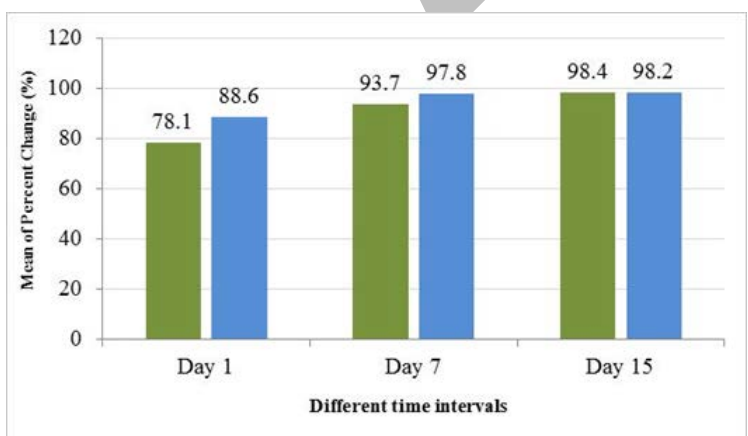

" Group I ReliaFIL LC $\quad$ Group II Riva LC

Figure (6): Comparison between fluoride recharge capacity for the two studied groups I (reliaFIL LC nano-hybrid composite) and group II (Riva LC RRGIs) at different time intervals.

\section{DISCUSSION}

A novel fluoride releasing nano-hybrid composite resin (ReliaFIL universal light cure composite) was recently introduced and it's characterized by better physical properties in addition to leachable fluoroaluminosilicate glass fillers as source of fluoride in its composition (19). The purpose of the present study was to compare the fluoride release capacity of fluoride this releasing nanohybrid composite resin (ReliaFIL light cure) with resin reinforced glass ionomer (Riva light cure) and the recharge capacity after treatment with topical fluoride varnish.

There are many methods that have been employed to estimate the amount of fluoride release such as spectrophotometry, ion chromatography, fluoride ionspecific electrodes and capillary electrophoresis. Ion specific electrode with an ion-analyser was used in this study because it is simple, inexpensive and does not require the use of complex laboratory equipment. Moreover, it gives an accurate and direct estimate of the free fluoride present in solution (20).

The results of this study showed that the fluoride released from reliaFIL LC in day 1 did not show any high initial release (burst effect) which was in agreement with Yap et al (20), Attar et al (21), Xu et al (22) and with Bansal et al (16) confirming all that fluoride containing resin composite exhibited a gradual, sustained pattern of fluoride release; therefore they lack the burst effect.

In addition, the release examined in day 7 was the highest among the three time intervals (day 1, 7 and day 15) which may be due to the mechanism of fluoride released from resin composite which is not an acid base reaction dependant. Instead it is thought to be a slow diffusion process mediated by an ability to allow water diffusion within the resin matrix. Itota et al (23) had also concluded that the fluoride released depends on the rate of water sorption of fluoride releasing restorative materials which is a diffusion controlled process and the segmental mobility of polymer chain within the matrix. As well, the fluoroaluminosilicate glass fillers added in the base of the resin matrix could have shut off the fluoride ions in the resin base making them difficult to be released initially however after 7 days more water diffusion may have led to increase the fluoride release.

Moreover, this study demonstrated that the median of fluoride release in day 15 was lower than release in day 7 which was in accordance with findings of Yab et al (20) and Mungara et al (15) that showed a decline in release from day 7 to day 15 . This could be explained by the fluoride release via an exchange mechanism in the direction of the lowest concentration as the existence of a concentration gradient is the driving force for fluoride release. It is expected that the fluoride release will decrease due to the diminishing of gradient as result of leaching out of fluoride from the materials. On the other hand, several studies $(25,16,24,26)$ had proposed that the pattern of fluoride release for fluoride releasing composite exhibited a high initial release from surface followed by a sharp decline after 2-3 days then stabilized sustained lower release.

In the current study, reliaFIL LC nano-hybrid composite released a measurable amount of fluoride during the whole study which may be attributed to the presence of leachable glass filler (fluoroaluminosilicate) as a source of fluoride incorporated into the composite. Itota et al (27) also reported that Unifil Flow "fluoride releasing composite containing fluoroaluminosilicate glass" had released a higher amount of fluoride than Heliomolar which is a fluoride releasing composite containing ytterbium 
trifluoride (YbF3). Also, it was reported by Naoum et al (28) that all fluoride releasing composite containing fluoroaluminosilicate glass demonstrated a good fluoride release ability. In contrary, Silva et al (29) noted that Tetric$\mathrm{N}$-Ceram (TNC) (fluoride releasing nano-hybrid composite containing YF3) showd no detectable amount of fluoride release during the experiment. This could be related to the low amount of fluoride incorporated TNC and the low solubility and permeability of sparingly salt (YbF3) used.

As for Riva light cure resin reinforced glass ionomer results, the median release in day 7 was higher than in day 1 which complied with Neelakantan et al (26) who illustrated that the 2-Hydroxyl Methacrylate (HEMA) present in RMGIs slowly absorbs water needed for the diffusion of fluoride ions causing gradual release of fluoride, thereby explaining the higher release through time intervals. This was in disagreement with other studies (25, $16,24,26)$ that revealed that fluoride release pattern of Riva LC appeared to be different than most of RMGIs that are characterized by a highly initial release at first day then a sharply decline occur till the 7th day. Those variations could be related to some of the factors related to the fluoride concentration in the set materials, size and composition of the inorganic filler, curing time and the inner-material porosity.

Comparing the results of the fluoride release from reliaFIL LC and Riva LC, it was concluded that Riva LC had a higher median of fluoride release at different time intervals. This finding is in agreement with $\mathrm{Xu}$ et al (22), Neelakantan et al (26), and Bansal et al (16) Silva et al (29). This could be due to the high solubility of $\mathrm{Ca}-\mathrm{Al}-\mathrm{F}$-silicate glass fillers in the resin-modified glass ionomers and their high porosity.

Professionally applied fluoride varnish is a common caries preventive measure used in pediatric dentistry (30). It was used to recharge the tested restorative materials to mimic the clinical situation. The ability of the topical fluoride agent for recharging a material is dependent on the dose, frequency, concentration and duration of application of this agent (24). The fluoride re-release that occurs immediately after recharge is induced by superficial effect of the topical fluoride. While during the subsequent days, fluoride release is attributed to the ability of topical fluoride varnish to be diffused through the materials' pores and to be stored until it would be re-released (34).

The results obtained in the present study showed that both reliaFIL LC nano-hybrid composite and Riva LC RRGIs were able to be recharged with topical fluoride with no significant difference between the two materials. While several studies $(28,32,33)$ were in disagreement with these findings; as the fluoride releasing composite displayed the least potential for fluoride recharge whereas the RMGIs displayed the greatest potential. This controversial finding may be attributed to the different fluoride filler system in reliaFIl LC nano-hybrid composite which is fluoroaluminosilicate leachable glass. The same glass filler in RMGIs is characterized by high solubility and high affinity to release fluoride. Thus, increasing the release and the recharge respectively due to the linear correlation between the release capacity and the recharge capacity.

By tracking the fluoride recharge pattern, it was found that all time intervals analysed showed a high recharge percent that sustained to be higher than the pre-exposure to ClinproTM white varnish which was also in disagreement with several studies (22,32-34). The recharge percent was higher after the first 24 hrs with sharply decline nearly to pre-exposure amount. This may be accredited to the use of ClinproTM white varnish containing functionalized tricalcium phosphate with 5\% sodium fluoride that was reported to have high and sustained fluoride release (35).

A possible limitation of the present study that its an invitro research that cannot replicate the natural oral conditions such as salivary flow characteristics, presence of plaque, difference in temperature and $\mathrm{pH}$ and the oral hygiene and dietary habits utilized by the patient. However, simulation of these conditions could give valuable informations.

Within the limitations of the present study, the first tested null hypothesis can be rejected as there was statistical significant difference in fluoride release between fluoride releasing nano-hybrid composite (reliaFIL LC) and Resin reinforced glass ionomer (Riva LC RRGI). The second tested null hypothesis was accepted, as there was no statistical significant difference between the two materials after fluoride varnish recharge.

As regard to the results of this study, reliaFIL light cure nano-hybrid composite could be considered a promising material that releases fluoride with the ability to be recharged by topically applied fluoride varnish. Hence, regular application of topical fluoride is essential to allow steady supply of fluoride ions, thereby, providing a balance of mechanical properties needed in loaded bearing restorations and fluoride release and recharge needed in high caries risk pediatric patients.

\section{CONCLUSION}

Based on the results of this study and its limitation, it could be concluded that fluoride releasing nano-hybrid composite (reliaFIL LC) had an acceptable fluoride release capacity and a recharge capacity comparable to resin reinforced glass ionomers.

\section{CONFLICT OF INTEREST}

The authors declare that they have no conflicts of interest.

\section{REFERENCES}

1-Edwina AM, Kidd OF. Essentials of Dental Caries. 4th ed. United Kingdom: Oxford University Press; 2016.

2-Frencken JE, Sharma P, Stenhouse L, Green D, Laverty D, Dietrich T. Global epidemiology of dental caries and severe periodontitis - a comprehensive review. J Clin Periodontol. 2017;44:S94-S105.

3-Bencze Z, Fraihat N, Varga O. Patent Landscape Analysis of Dental Caries in Primary Teeth. J Environ Res Public Health. 2019;16. pii: E2220.

4-Brouwer F, Askar H, Paris S, Schwendicke F. Detecting Secondary Caries Lesions: A Systematic Review and Metaanalysis. Dent Res. 2016;95:143-51.

5-Hollanders ACC, Kuper NK, Opdam NJM, Huysmans MCDNJM. Preventive dentistry 5. Secondary caries. Ned Tijdschr Tandheelkd. 2017;124:257-63.

6-Innes NPT, Chu CH, Fontana M, Lo ECM, Thomson WM, Uribe S, et al. A Century of Change towards Prevention and Minimal Intervention in Cariology. J Dent Res. 2019;98:611-7.

7-Łukomska-Szymańska M, Zarzycka B, Grzegorczyk J, Sokołowski K, Półtorak K, Sokołowski J, et al. 
Antibacterial Properties of Calcium Fluoride-Based Composite Materials: In Vitro Study. Biomed Res Int. 2016;2016:1048320.

8-Chu CH, Mei ML, Lo EC. Use of fluorides in dental caries management. Gen Dent. 2010;58:37-43.

9-McCoy T. Fluoride-Releasing Restorative Materials. J Vet Dent. 2015;32:196-7.

10-Baig MS, Fleming GJ. Conventional glass-ionomer materials: A review of the developments in glass powder, polyacid liquid and the strategies of reinforcement. J Dent. 2015;43:897-912.

11-Chisini LA, Collares K, Cademartori MG, de Oliveira LJC, Conde MCM, Demarco FF, et al. Restorations in primary teeth: a systematic review on survival and reasons for failures. Int J Paediatr Dent. 2018;28:123-39.

12-Davis HB, Gwinner F, Mitchell JC, Ferracane JL. Ion release from, and fluoride recharge of a composite with a fluoride-containing bioactive glass. Dent Mater. 2014;30:1187-94.

13-Bhardwaj A, Misuriya A, Maroli S, Manjula S, Singh AK. Nanotechnology in dentistry: Present and future. J Int Oral Health. 2014;6:121-6.

14-Jacob C. Statistical power analysis for the behavioral sciences. 2nd ed. Hillsdale, NJ: Lawrence Erlbaum; 1988.

15-Mungara J, Philip J, Joseph E, Rajendran S, Elangovan A, Selvaraju G. Comparative evaluation of fluoride release and recharge of pre-reacted glass ionomer composite and nanoionomeric glass ionomer with daily fluoride exposure: an in vitro study. J Indian Soc Pedod Prev Dent. 2013;31:234-9.

16-Bansal R, Bansal T. A comparative evaluation of the amount of fluoride release and re-release after recharging from aesthetic restorative materials: An in vitro study. J Clin Diagn Res. 2015;9:11-4.

17-Miller D. Ion-Selective Electrode Determination of Fluoride Ion. Chem Lab Manual 2011;321:23-7.

18-IBM Corp. IBM SPSS Statistics for Windows, Version 25.0. Armonk, NY: IBM Corp; Released 2017.

19-Advanced Healthcare Ltd. AHL Brand Catalogue. Tonbridge, UK: 1986. Available at: http://www.ahl.uk.com/images/pdf/AHL-2017Catalogue.pdf

20-Yap AU, Tham SY, Zhu LY, Lee HK. Short-term fluoride release from various aesthetic restorative materials. Oper Dent. 2002;27:259-65.

21-Attar N, Turgut MD. Fluoride release and uptake capacities of fluoride-releasing restorative materials. Oper Dent. 2003;28:395-402.

22-Xu X, Burgess JO. Compressive strength, fluoride release and recharge of fluoride-releasing materials. Biomat. 2003;24:2451-61.

23-Itota T, Carrick TE, Rusby S, Al-Naimi OT, Yoshiyama M, McCabe JF. Determination of fluoride ions released from resin-based dental materials using ion-selective electrode and ion chromatograph. J Dent. 2004;32:117-22.
24-Wiegand A, Buchalla W, Attin T. Review on fluoridereleasing restorative materials--fluoride release and uptake characteristics, antibacterial activity and influence on caries formation. Dent Mater. 2007;23:343-62.

25-Nigam AG, Murthy R, Pandey R. Estimation of Fluoride Release from Various Dental Materials in Different Media-An In Vitro Study. Int J Clin Pediatr Dent. 2009;2:1-8.

26-Neelakantan P, John S, Anand S, Sureshbabu N, Subbarao C. Fluoride release from a new glass-ionomer cement. Oper Dent. 2011;36:80-5.

27-Itota T, Al-Naimi OT, Carrick TE, Yoshiyama M, McCabe JF. Fluoride release and neutralizing effect by resin-based materials. Oper Dent. 2005;30:522-7.

28-Naoum S, Ellakwa A, Martin F, Swain M. Fluoride release, recharge and mechanical property stability of various fluoride-containing resin composites. Oper Dent. 2011;36:422-32.

29-Silva RAB, Santos FRR, Spadaro ACC, Polizello ACM, De Rossi A, Moreira MR, Nelson-Filho P. Profile of fluoride release from a nanohybrid composite resin. Dent 3000. 2015;3:1-4.

30-Marinho VC, Worthington HV, Walsh T, Clarkson JE. Fluoride varnishes for preventing dental caries in children and adolescents. Cochrane Database Syst Rev. 2013:Cd002279.

31-Weyant RJ, Tracy SL, Anselmo TT, Beltran-Aguilar ED, Donly KJ, Frese WA, et al. Topical fluoride for caries prevention: executive summary of the updated clinical recommendations and supporting systematic review. J Am Dent Assoc. 2013;144:1279-91.

32-Dionysopoulos D. The effect of fluoride-releasing restorative materials on inhibition of secondary caries formation. Fluoride. 2014;47:258-65.

33-Dasgupta S, Saraswathi MV, Somayaji K, Pentapati KC, Shetty P. Comparative evaluation of fluoride release and recharge potential of novel and traditional fluoridereleasing restorative materials: An in vitro study. J Conserv Dent. 2018;21:622-6.

34-Preston AJ, Agalamanyi EA, Higham SM, Mair LH. The recharge of esthetic dental restorative materials with fluoride in vitro-two years' results. Dent Mater. 2003;19:327.

35-Rirattanapong $\mathrm{P}$, Vongsavan K, Saengsirinavin C, Jantarakam N. A 3-month study of fluoride release from different calcium phosphate fluoride varnishes on primary teeth. Southeast Asian J Trop Med Public Health. 2016;47:1098-104. 